\section{Importancia de los recursos audiovisuales en los docentes y estudiantes durante la Pandemia por COVID-19}

Importance of Audiovisual Resources in Teacher and Students During the COVID-19 Pandemic

Luis Alberto Chávez Ramos, Adelaida Maria del Carmen Hualpa Flores, Eva Luis Paredes, Edgar Hugo Vásquez Condezo

\section{RESUMEN}

La presencia del COVID-19 produjo cambios drásticos en el sector educativo, teniendo que recurrir a nuevas estrategias y metodologías para la enseñanza mediante el uso de recursos audiovisuales gracias a las Tecnologías de la Información y Comunicación. El presente artículo tiene como objetivo conocer la importancia del uso de los videos en la etapa de enseñanza y aprendizaje virtual en tiempos de pandemia. La investigación tuvo como base la revisión documental de artículos científicos en revistas de alto impacto, desde un enfoque cualitativo y de nivel descriptivo. Se evidenció que YouTube se ha convertido en un ambiente perfecto para docentes, siendo una fuente motivadora para una orientación audiovisual multidisciplinaria en los estudiantes. Durante la pandemia se intensificó la utilidad del video en el aula invertida como método innovador de aprendizaje resaltando la motivación intrínseca del estudiante. Además, se resalta una nueva generación de maestros y estudiantes prosumidores en la elaboración de contenidos audiovisuales temáticos, desarrollando competencias digitales en bien de la enseñanza y el aprendizaje.

Palabras clave: Audiovisuales; videos; educación; pandemia; COVID-19

\section{ABSTRACT}

The presence of COVID-19 produced drastic changes in the education sector, having to resort to new strategies and methodologies for teaching through the use of audiovisual resources thanks to Information and Communication Technologies. This article aims to know the importance of the use of videos in the teaching and virtual learning stage in times of pandemics. The research was based on the documentary review of scientific articles in high-impact journals, from a qualitative and descriptive level approach. It was evidenced that YouTube has become a perfect environment for teachers, being a motivating source for a multidisciplinary audiovisual orientation in students. During the pandemic, the usefulness of video in the inverted classroom as an innovative method of learning intensified, highlighting the intrinsic motivation of the student. In addition, it highlights a new generation of teachers and students prosumers in the elaboration of thematic audiovisual content, developing digital skills for the sake of teaching and learning.

Keywords: Audiovisual; videos; education; pandemic; COVID-19
RELIGACIÓN

REVISTA DE CIENCIAS SOCIALES Y HUMANIDADES JOURNAL OF SOCIAL SCIENCES AND HUMANITIES
REVISTA DE CENCIAS SOCIAIS E HUMANAS

\section{INFORMACIÓN:}

https://doi.org/10.46652/rgn.v6izo.833

ISSN 2477-9083

Vol. 6 No. $30,2021$. e210833

Quito, Ecuador

Enviado: 30 agosto 2021

Aceptado: 04 octubre 2021

Publicado: 29 octubre 2021

Publicación Continua

Sección General | Peer Reviewed

\section{cc) $\$$} open 2 access

\section{AUTORES:}

(i) Luis Alberto Chávez Ramos. Universidad César Vallejo - Perú Ichavezra@ucvvirtual.edu.pe (iD) Adelaida Hualpa Flores. Universidad César Vallejo - Perú ahualpaf@ucvvirtual.edu.pe (iD) Eva Luis Paredes

Universidad César Vallejo - Perú eluisp@ucvvirtual.edu.pe

(D) Edgar Hugo Vásquez Condezo. Universidad César Vallejo - Perú ehvasquezv@ucv.edu.pe

\section{CONFLICTO DE INTERESES}

Los autores declaran que no existe conflicto de interés posible.

FINANCIAMIENTO

No existió asistencia financiera de partes externas al presente artículo.

NOTA

El artículo no se desprende de investigaciones previas.

ENTIDAD EDITORA CICSHYAL 


\section{Introducción}

La irrupción del SARS-CoV-2 encendía las alarmas a nivel mundial con la presencia de una nueva pandemia en el presente siglo XXI. En el Perú, 15 de marzo de 2020 el presidente en turno Martín Vizcarra, estableció el estado de emergencia en todo el territorio nacional por la presencia del primer caso de COVID-19. Mientras tanto y ante la necesidad de no dejar a los estudiantes sin educación durante el estado de emergencia sanitaria, el 6 de abril del mismo año el Ministerio de educación dio inicio al año escolar en instituciones públicas mediante el uso de la plataforma "Aprendo en casa" (Sobeida y Navarro, 2021) cuyos contenidos fueron difundidos a través de la web, radio y televisión.

El uso de los medios audiovisuales en la educación ha mantenido una estrecha relación, posibilitando al estudiante conocer nuevas experiencias de conocimiento y generando un desarrollo cognoscitivo, teniendo como vías de percepción la vista y el oído como principales receptores de información ante un material audiovisual. En tal sentido, recursos audiovisuales como las cámaras de video, la televisión, proyectores, entre otras, han encontrado un sitial importante en la red, convirtiendo este espacio en un lugar de privilegio para los nativos digitales que mediante diversas plataformas y aplicativos digitales, encuentran una motivación intrínseca por visualizar y a la vez crear contenidos que sean complemento de su aprendizaje, siempre bajo la supervisión del docente de la materia.

Ante la aparición repentina de la pandemia del COVID-19, no permitió a tiempo el desarrollo de estrategias para la capacitación y actualización en los maestros y estudiantes en el manejo de entornos virtuales y herramientas tecnológicas con fines pedagógicos. Esto sin dejar de mencionar que la educación a distancia virtual ha estado presente antes de la llegada de la pandemia y que lastimosamente no estuvo muy bien implementada en la educación básica o superior a pesar del avance tecnológico en el desarrollo en entornos virtuales de aprendizaje. Las tecnologías de la información y comunicación (TIC) se han convertido en los aliados estratégicos para la enseñanza virtual, sobre todo en el uso de diversos medios audiovisuales que despertaron el interés en su aplicación por parte de maestros y estudiantes.

En relación con el origen de los productos audiovisuales. A finales del siglo XIX se consolidó la creación de un aparato que pudiera plasmar imágenes en movimiento sobre un soporte; el cinematógrafo, dio inicio al nacimiento del cine de la mano de los hermanos Lumiere. Con el transcurrir del tiempo las imágenes en movimiento se convertirían en un aliado indispensable en la educación, los docentes fueron aplicando contenidos audiovisuales como apoyo en el proceso de aprendizaje, siendo aplicado en sus inicios en sesiones complejas (Barros, 2015) en ese sentido, el uso de estos contenidos audiovisuales buscó una motivación especial para atraer visualmente al estudiante y por ende, motivar su desarrollo cognoscitivo en todos los niveles, sobre todo desde la etapa inicial de formación académica.

Con respecto a la teoría de la ecología de los medios, las personas se relacionan con otras a través de medios tecnológicos (Sun y Zhong, 2020) y que han sido asequibles en el hogar, pero también en el aula, en este último, ha implicado un nuevo sistema educativo empleando recursos como las fotografías, los audios, los videos; una estrategia comunicativa que basado en el uso de las nuevas tecnologías ha servido en el proceso de enseñanza y aprendizaje; y que se han convertido en una herramienta importante en el sector educación durante la práctica docente. (Barros, 2015) los medios de comunicación han ocupado un lugar fundamental como herramienta de aprendizaje donde los medios tradicionales de comunicación como la radio, televisión, prensa y el internet han ayudado a la persona y en especial al estudiante en formación a ser reflexivo, 
aprende a reflexionar y analizar todo tipo de contenido que es vertido a través de los medios. En ese sentido, los recursos audiovisuales se han convertido en un aliado estratégico para la educación presencial como virtual en estos últimos años en pandemia.

El uso obligatorio de la tecnología en el sector educación experimentó una nueva dinámica de enseñanza y aprendizaje, nuevos términos se asociaban a la vez durante la pandemia, lo sincrónico y lo asincrónico. Si bien es cierto ambas son producto del uso de entornos virtuales de aprendizaje, existieron otros factores como el analfabetismo y la brecha digital en el campo educativo que impidieron su evolución en el campo educativo. El uso de diversos recursos audiovisuales empezó a tomar mayor importancia como estrategia única de contacto y de enseñanza no presencial, dando paso al uso de plataformas como Meet, Zoom, Skype, Microsoft Teams, etc., (Bakhronova, 2021), convirtiéndose en los principales medios audiovisuales para las escuelas, donde la interacción entre maestro y estudiantes se convertiría en una nueva experiencia de educación desde la virtualidad.

Como expresa Ezquerra (2018) el acelerado cambio tecnológico no ha permitido que muchos docentes se hayan formado en el uso de medios tecnológicos. En ese sentido, la brecha digital que existe en la plana docente y por desconocimiento del uso de plataformas digitales nos indica que estamos en una nueva generación de docentes que ha empezado a incorporar piezas audiovisuales con el fin de complementar la información temática durante su proceso de enseñanza. Esta ruptura de paradigmas en el uso de recursos audiovisuales pasó de ser de un nivel aficionado, a ser usado de manera masiva durante los procesos de enseñanza, convirtiendo así a los recursos audiovisuales como una utilidad didáctica en el autoaprendizaje del estudiante (Marcos, 2020).

En la actualidad, existen canales de video que son utilizados como recursos para compartir contenidos audiovisuales, el más requerido por docentes y estudiantes es YouTube, por ser de libre acceso y utilizados de manera estratégica para consumir información y producir nuevos contenidos, constituyendo así el nacimiento de maestros prosumidores de acuerdo con las temáticas propuestas para alcanzar los objetivos del año académico. Además, mediante el uso de estos materiales audiovisuales se puede establecer un orden invertido o lo denominado clase invertida en donde el estudiante pueda visualizar los contenidos para luego ser utilizados a manera de debate en clase, tal como lo señala Climent y Vidal (2021).

En los últimos años las instituciones educativas han experimentado cambios estratégicos haciendo énfasis en la innovación. "Desde la concepción conductista, se percibe a las TIC como estímulos que pueden favorecer el desarrollo de respuestas específicas mediante el refuerzo, destacando como tecnología la enseñanza programada" (Cabero, 2015, p. 188) esto quiere decir que los espacios virtuales han logrado posicionarse dentro del marco académico haciendo que la educación en todos los niveles empiece a adaptarse y encontrar un nuevo proceso de aprendizaje virtual. Marcos y Moreno (2020) dan a conocer en su disertación que los recursos audiovisuales suelen ser una innovadora y creativa forma de llevar conocimiento en especial para el público joven cuyo consumo de productos audiovisuales desde películas hasta spots publicitarios genera nuevos contenidos conceptuales como actitudinales. Del mismo modo, Goian et al. (2021) menciona que los productos audiovisuales vertidos a través del cine, televisión e internet han servido como un componente tecnológico educativo durante la crisis del COVID-19.

En este contexto de pandemia se han propuesto estrategias de aprendizaje que gracias al uso de las tecnologías han encontrado un espacio para el uso de materiales audiovisuales como su aplicación en Flipped Classroom o clase invertida, considerar a YouTube como plataforma de 
consulta audiovisual y el desarrollo de capacidades para crear contenidos audiovisuales. Por este motivo, el uso del video en el desarrollo de las actividades académicas debería ser clave para desarrollar un aprendizaje autónomo y significativo.

Por tal motivo, el presente artículo tiene como objetivo conocer la importancia del uso de los videos de manera sincrónica y asincrónica en el proceso de enseñanza durante la etapa de pandemia de COVID-19.

\section{Metodología}

El presente artículo se realizó en base a una revisión documental de artículos científicos en bases de datos como Scopus, Scielo, Latindex, entre otras y relacionados en el uso de recursos audiovisuales en la educación durante la pandemia, producto de la presencia del COVID-19; haciendo énfasis en la experiencia del uso de YouTube, la clase invertida y la formación de prosumidores temáticos. Para la búsqueda de información se utilizaron operadores booleanos como "and" y "or". Los artículos hallados tuvieron como un tiempo máximo de antigüedad de dos años que coinciden con el periodo de la pandemia de COVID-19, los cuales fueron sometidos a criterios de inclusión y exclusión para la realización del presente estudio, haciendo énfasis en el uso de YouTube en el aula virtual, la aplicación de piezas audiovisuales en el aula invertida y a la creación de contenidos audiovisuales por parte de los prosumidores.

\section{Desarrollo}

Ante el inesperado cierre del desarrollo de actividades escolares producto de la presencia del COVID-19 en el Perú, el estado empezó una nueva tarea en el uso de una plataforma integral para el apoyo de contenidos audiovisuales, haciendo uso de medios tradicionales como radio, televisión e internet. Este último, ha tenido un gran impacto en esta migración obligatoria a esta no tan reciente modalidad virtual, que tuvo como antecesor a la educación por correspondencia siendo este el primer modelo de educación a distancia, donde el maestro y estudiante experimentaron una etapa en la enseñanza y aprendizaje rompiendo las barreras de espacio y tiempo.

Aplicar el uso del video durante la etapa de enseñanza y aprendizaje es un procedimiento valioso para el desarrollo lingüístico y para el logro de competencias digitales durante la formación del estudiante (Climent y Vidal, 2021). El uso de video profesional en la enseñanza dejó de serlo desde la aparición de YouTube en el 2005, en el cual toda persona podía generar nuevos contenidos audiovisuales con fines diversos y compartirlo a través de esta red social. Este recurso a la vez incentiva que los estudiantes puedan experimentar el aprendizaje en línea, a conocer nuevos procedimientos de aprendizaje informal de manera entretenida con incidencia en el autoaprendizaje mediante la visualización de contenidos temáticos (Temban et al., 2021).

El uso de las redes sociales virtuales durante la crisis sanitaria producto del COVID-19, han sido vitales para el intercambio de contenidos y se consideran como una poderosa herramienta comunicacional de soporte para el aprendizaje de los estudiantes que hacen uso de diversas plataformas para la interacción sincrónica y asincrónica (Goian et al., 2021). De esta manera, el estudiante ha incorporado las TIC como una herramienta fundamental para el intercambio de material multimedia y audiovisual como un recurso interactivo donde ha aprendido a obtener, procesar y crear información. Durante el estado de pandemia, los medios audiovisuales fueron ocupando un lugar importante para la enseñanza, ya no era solo las presentaciones en diapositivas, fotografías o documentos en línea. La incorporación de técnicas discursivas dio pie a intensificar la visualización y creación de contenidos audiovisuales como estrategia didáctica por parte del docente. 
En ese sentido, partiremos definiendo de manera conceptual a YouTube, Clase invertida y Prosumidor desde el punto de vista de autores que elaboraron artículos científicos en esta etapa de pandemia y que de una manera inductiva podamos entender cómo en la actualidad se definen estos conceptos que nos ayudarán a entender la proximidad del uso de los videos como parte de las estrategias para la enseñanza y aprendizaje de los estudiantes en los diversos niveles en educación.

Tabla 1: Conceptos teóricos

\begin{tabular}{|c|l|l|}
\hline RECURSOS AUDIOVISUALES & \multicolumn{1}{|c|}{ AUTOR } & \multicolumn{1}{c|}{ CONCEPTO } \\
\hline YouTube & (Maraza et al., 2020) & $\begin{array}{l}\text { YouTube es una plataforma interactiva gratuita, permi- } \\
\text { te reproducir videos con diversos contenidos ilimitada- } \\
\text { mente, en el aspecto educativo el estudiante va cons- } \\
\text { truyendo su propia información debido a la percepción } \\
\text { de elementos multimedia durante su visualización. }\end{array}$ \\
\hline Clase invertida & $\begin{array}{l}\text { Estrategia de aprendizaje gracias al uso de las TIC, don- } \\
\text { de el estudiante fuera del claustro escolar utiliza los } \\
\text { diversos recursos didácticos para acceder al primer } \\
\text { contacto temático antes de clase, contribuyendo al } \\
\text { autoaprendizaje, rompiendo las barreras de tiempo y } \\
\text { espacio en la educación virtual. }\end{array}$ \\
\hline Prosumidor & $\begin{array}{l}\text { Establece la unión de los vocablos: Productor y Consu- } \\
\text { midor. En la práctica se refiere a una persona que con- } \\
\text { sume y produce información como parte de las diná- } \\
\text { micas sociales, siendo visible a gran escala en internet. }\end{array}$ \\
\hline
\end{tabular}

Fuente: Elaboración propia.

\subsection{YouTube}

La presencia del COVID-19 dio como consecuencia que la plana docente y estudiantil se refugiaran en la etapa de cuarentena; en ese sentido, las redes sociales se convirtieron en el principal medio de información y comunicación, teniendo como aliados para ello a WhatsApp, Facebook, Twitter, Instagram y YouTube. Este último, se transformó en la plataforma idónea para ser utilizada por maestros con el objetivo de transmitir contenidos debido a su alto consumo, de una manera estratégica de difusión masiva durante la etapa de confinamiento (Ramírez et al., 2020) y que ha logrado encontrar un aprendizaje significativo al integrar como propuesta didáctica a esta red social en favor de la educación (Del Valle-Ramón et al., 2020).

Tras su creación en el año 2005, YouTube se convertiría en una plataforma de almacenamiento de videos de acceso abierto y gratuito, pasado el tiempo se ha convertido en el principal repositorio audiovisual y que, gracias a su afiliación con Google, toda persona puede crear su propio canal de YouTube con tan solo contar con una cuenta en Gmail. Desde sus inicios, los contenidos fueron de género de entretenimiento, pero también se convirtió en una plataforma importante para compartir información y que este, a la vez, generará una interacción con la persona mediante las suscripciones, las visualizaciones, los comentarios, el compartir el material audiovisual por medio del hipertexto en otras plataformas, en páginas web, en redes sociales, entre otros.

Desde la aparición de la plataforma de YouTube se abrieron muchas puertas para personas y empresas que deseaban compartir sus experiencias, productos y servicios; la educación no fue la excepción "Hoy en día es una de las páginas web más usadas en todo el mundo y la mayoría de los docentes la utiliza para obtener recursos audiovisuales” (Pattier, 2020, p. 86). 
Lozano-Blasco et al., (2021) manifiesta que durante la pandemia producto del COVID-19, YouTube se convirtió en una herramienta importante no solo para el área de entretenimiento, también para el sector educativo debido a las medidas adoptadas por los distintos gobiernos en disminuir radicalmente la enseñanza presencial y trasladarla a una modalidad en línea. En ese sentido, muchos niños haciendo uso de sus habilidades psicomotoras de las manos utilizaron dispositivos móviles como Smartphones y tabletas que han sido los principales soportes para el acceso a internet, pero, sobre todo al uso de la aplicación de YouTube, donde los infantes se sintieron atraídos por diversos contenidos educativos como videos musicales, cuentos, etc. dejando de lado la socialización e interacción con otros niños durante la pandemia.

Temban et al. (2021) señala que el uso del YouTube se convirtió en una estrategia de enseñanza siendo utilizada hoy en día por diversas instituciones que imparten conocimiento en todos sus niveles, generando contenidos educativos con el fin de complementar la información en los diversos tópicos usados durante las sesiones telemáticas. Por otro lado, también cataloga a esta plataforma como el Rey de los videos y que ha sido de soporte importante para la formación de niños en su aprendizaje informal durante esta crisis mundial generada por la pandemia y que recayó en una versión para este segmento de población denominada YouTube Kids que, de acuerdo con los contenidos, está contribuyendo al desarrollo de conocimiento y entretenimiento de los menores en etapa escolar.

En palabras de Barredo et al. (2021), gracias a los avances tecnológicos YouTube se ha convertido en una de las plataformas más populares en los últimos años y que está siendo aprovechado por la comunidad juvenil estudiantil, esto, gracias a la expansión de los dispositivos móviles que permite el valor agregado en la visualización de contenidos sobre todo al referir contenidos desde un punto de vista académico. Como expresa Climent y Vidal (2021) nos demuestra que YouTube se ha convertido en una fuente de consulta académica muy importante para los estudiantes y que su uso cotidiano crea un nuevo espacio virtual donde la ausencia del maestro en un momento asincrónico es suplida por videotutoriales, documentales, series, entrevistas, etc.

A juicio de Neumann y Herodotou (2020), YouTube es utilizado como estrategia de autoaprendizaje ya que proporciona de información útil para el estudiante, pero, que este recurso debe contar con el monitoreo de padres y maestros para garantizar la eficacia de los contenidos vertidos, que sean los adecuados y que proporcionen conocimiento sobre el estudiante quien visualiza las diversas piezas audiovisuales, sobre todo en niños que empiezan a hacer uso de esta plataforma de una manera meramente informativa.

\subsection{Clase invertida}

En palabras de Climent y Vidal (2021) la clase invertida o conocida como Flipped Classroom se ha convertido en una metodología básica en estos tiempos. Consiste en hacer uso de materiales audiovisuales para que el estudiante pueda observar los diversos contenidos para ser utilizados posteriormente en las sesiones sincrónicas. En ese sentido, los docentes emplearon una nueva estrategia para complementar la información que no se podía realizar de manera presencial, para ello, muchos optaron por contenidos temáticos que se encuentran en las diversas plataformas videos, siendo la más importante y relevante YouTube. El presente modelo pedagógico ofrece al estudiante diversos materiales para ser usados fuera del aula presencial o sincrónica, desarrollando nuevas prácticas y habilidades autónomas (Alarcón, 2021).

Para Hernándezy Martinez (2021) el uso de los videos educativos están siendo empleados con mucha frecuencia para realizar clase invertida y guiando al estudiante a un sistema de autoaprendizaje 
previo a la presencia en el aula virtual o de manera sincrónica, estableciendo nuevos criterios durante el proceso de enseñanza y aprendizaje. La aplicación del aula invertida ha dado paso a la creación de los entornos virtuales de aprendizaje (EVA) (Salas-Rueda, 2021); en estos entornos, los estudiantes han recurrido al uso de contenidos multimedia y recursos audiovisuales de sus asignaturas en cualquier momento y lugar, ayudando al escolar en su personalización en la etapa de aprendizaje. Agrega que el personal docente se adaptó al cambio, creando material audiovisual en las diversas sesiones telemáticas.

El aula invertida se ha convertido en un modelo innovador, ocupando el espacio del hogar en un espacio de aprendizaje tal como lo haría en un aula de clase (Avila y Tapia, 2020). Durante la pandemia esta metodología de enseñanza y aprendizaje presentó buenos resultados en todos los niveles educativos, donde el estudiante valoró el autoaprendizaje a través de videos desarrollando un aprendizaje significativo. Esto nos permite incentivar a los estudiantes a ser los protagonistas del desarrollo de contenidos a través de las sesiones virtuales (Janssen, 2020). Actualmente, y en esta etapa de pandemia, se aceleró la práctica del aula invertida gracias a la tecnología, transformando el modo de la realización de las actividades escolares por ser ubicua e incentiva al trabajo colaborativo, utilizando el tiempo fuera del aula para visualizar, debatir y consensuar contenidos relacionados a las diversas temáticas consignadas en el sílabo del curso.

Los recursos audiovisuales cumplen una labor fundamental en la aplicación del aula invertida, gracias a esta, facilita el traslado de información hacia el estudiante, fomentándolo de manera activa en el proceso de aprendizaje. Campillo y Miralles (2021) corroboran el aumento del aprendizaje invertido gracias a los diversos escenarios virtuales que han llevado a la expansión de e-learning durante la pandemia del COVID-19 y que ha significado el aumento de niveles de motivación, progreso y percepción positiva al adquirir competencias digitales. Collado et al., (2021) revela resultados positivos gracias a las sesiones de aula invertida gracias a la variedad de materiales de archivo, recursos audiovisuales, etc. y que ha permitido mejorar la metodología educativa dejando de ser un complemento para convertirse en esencial durante el confinamiento.

\subsection{Prosumidores}

Este término fue acuñado por Alvin Toffler en su libro La Tercera Ola y hace referencia a toda persona que, mediante el consumo de información a través de los medios, produce un contenido subjetivo y que es utilizado como propuesta de un nuevo concepto. Desde el punto de vista de Barredo et al. (2021), ser Prosumer hoy en día es la capacidad que tiene la persona para analizar e interpretar un contenido a manera de hermenéutica, y que gracias a las TIC se han intensificado la creación de nuevos contenidos que parten desde las lecturas en diarios, libros hasta fotografías, videos, etc., y que esta interrelación de la información, la tecnología y la producción de contenidos, han constituido elementos importantes para que los estudiantes puedan crear argumentos válidos durante su formación académica.

Para los docentes, el uso de imágenes estáticas ya no era muy atractivo para los estudiantes, incorporar el uso de materiales audiovisuales demostró un procedimiento válido los cuales tuvieron como resultado la atención del estudiante (Manson, 2019). Este procedimiento del uso de elementos visuales y audiovisuales dio pie a que los maestros puedan crear nuevos contenidos basados en materiales ya elaborados e incorporados durante las sesiones de aprendizaje sincrónico o asincrónico. Además, el autor agrega que el alumnado actual está inmerso en una sociedad virtual que está rodeada de data, que esto incentiva por medio de la tecnología a la creación de nuevos contenidos de modo audiovisual y que poco a poco se ha ido integrando en la enseñanza. 
Para los nativos digitales es sencillo elaborar un material audiovisual. Hoy en día, y gracias a las redes sociales, es permitido que el uso de estos contenidos pueda ser difundido como parte de la expresión y relación con la comunidad virtual. En una etapa primigenia, los jóvenes divulgan contenidos irrelevantes dirigidos a sus pares donde podemos observar que el grado de creatividad se va constituyendo como una capacidad del estudiante con fines de comunicación e información.

Para el nacimiento de un Prosumidor es necesario que este aprenda a informarse; en ese sentido Triviño et al. (2021) menciona que YouTube es la plataforma de videos más buscada al navegar en internet y que los jóvenes destacan su importancia por encontrar información relevante que ayuda a la construcción de nuevos conocimientos durante un proceso de investigación. Así, el autor precisa que en diversos momentos de la creación de contenidos el estudiante se convierte en un Prosumidor, desarrollando habilidades digitales con fines académicos, y que se han visto intensificados durante la etapa de confinamiento producto de la pandemia mundial del COVID-19.

En tal sentido, Vasilchenko et al. (2020) sostiene que los estudiantes no estuvieron preparados para asumir el rol de prosumidores. Pero, desde un enfoque pedagógico, tanto los instructores y los estudiantes se han ido adaptando al cambio, asumiendo estrategias de enseñanza y aprendizaje no tradicional, donde el consumo y producción de contenidos se ha visto transformada en componentes didácticos como la elaboración de videos y creación de material multimedia de las manos de los propios estudiantes. La práctica prosumidora en esta etapa de pandemia ha recaído en la producción audiovisual de contenidos por parte de docentes y estudiantes en las diversas plataformas virtuales. De esta forma se incentiva al desarrollo de competencias y habilidades en el proceso de enseñanza y aprendizaje aprovechando el conocimiento tecnológico de los denominados "nativos digitales".

\section{Conclusión}

Tras la revisión de los artículos podemos concluir que los recursos audiovisuales han tenido un protagonismo excepcional durante el tiempo de pandemia producto del COVID-19. Los autores coinciden en sus investigaciones que la aplicación de piezas audiovisuales en los entornos virtuales ha colaborado con nuevas estrategias disruptivas en el proceso de enseñanza y aprendizaje.

La revisión resaltó el uso de YouTube como una plataforma importante para ser usado en las sesiones sincrónicas y asincrónicas durante la pandemia, convirtiéndose en un ambiente perfecto para docentes, siendo una fuente motivadora para una orientación audiovisual multidisciplinaria en los estudiantes. Los contenidos en esta red social han permitido que los estudiantes no solo puedan encontrar entretenimiento en ella, sino también una forma en la que pueden auto aprender y disponiendo del material audiovisual en cualquier momento. Esta herramienta de aprendizaje informal pasó de ser de entretenimiento, a un espacio ideal para consumir información relevante para anticipar o complementar información en el aula de clase.

También, se intensificó el uso del aula invertida, situación que dio al estudiante la oportunidad de conocer la temática desde cualquier dispositivo móvil u ordenador con la finalidad de que el contenido pueda ser debatido en el aula virtual de manera sincrónica. Si bien esta estrategia nació como estrategia de enseñanza para aprender diversos idiomas, ahora en tiempos de pandemia, ha logrado ser un método recomendado para anticiparse a las sesiones telemáticas, donde el estudiante cobra un protagonismo gracias al conocimiento adquirido antes de la sesión programada por el docente. Gracias a esta estrategia los estudiantes reflexionan y crean sus propios conceptos para ser debatidos con sus pares en el aula virtual. 
En consecuencia, se dio paso a la generación de nuevos prosumidores viéndose reflejada en la creación de contenidos audiovisuales por docentes que en su mayoría son denominados como migrantes digitales y que han ido adaptándose al cambio producto de la pandemia. Por otro lado, los denominados nativos digitales han incurrido en la creación de material audiovisual para plasmar las diversas actividades académicas, que mediante el proceso de grabación y edición de videos han logrado crear contenidos educativos para complementar un eje temático de acuerdo con el desarrollo de la asignatura.

Tras el presente trabajo de investigación se recomienda en futuras investigaciones conocer el impacto en el uso del contenido audiovisual de YouTube y si este influye en su rendimiento académico. El evaluar los resultados de la aplicación del aula invertida a través de la retroalimentación en clase. Además, investigar la motivación intrínseca en los estudiantes para crear contenidos audiovisuales con fines educativos.

\section{REFERENCIAS}

Alarcón, D. (2021). El aula invertida como estrategia de aprendizaje. CONRADO, 17(80), 152-157. https:// conrado.ucf.edu.cu/index.php/conrado/article/view/1823

Alonzo, R. (2019). Sobre el uso de los conceptos prosumer y emirec para estudios sobre comunicación mediada por internet. Sphera Publica, 1(19), 2-23. http://sphera.ucam.edu/index.php/sphera-01/article/ view/361/14141441

Avila, A. A. C., \& Tapia, C. E. C. (2020). Systematic review of the flipped classroom in Ecuador: approach to the state of the art. Estudios Pedagogicos, 46(3), 45-58. https://doi.org/10.4067/So71807052020000300045

Bakhronova, D. (2021). COVID-19y Educación Superior. La mundialización de la educación en línea. Religación. Revista De Ciencias Sociales Y Humanidades, 6(28), 62-69. https://doi.org/10.46652/rgn.v6i28.809

Barredo, D., Pérez Rodríguez, A., \& Aguaded, I. (2021). La influencia de la formación recibida en la realización y la difusión de contenidos audiovisuales en los adolescentes de Iberoamérica. Revista Letral, 26, 218-237. https://doi.org/10.30827/rl.voi26.17725

Cabero Almenara, J., \& Del Carmen Llorente Cejudo, M. (2015). Tecnologías de la Información y la Comunicación (TIC): escenarios formativos y teorías del aprendizaje. Revista Lasallista de Investigacion, 12(2), 186-193. https://doi.org/10.22507/rli.v12n2a19

Campillo-Ferrer, J. M., \& Miralles-Martínez, P. (2021). Effectiveness of the flipped classroom model on students' self-reported motivation and learning during the COVID-19 pandemic. Humanities and Social Sciences Communications, 8(1). https://doi.org/10.1057/s41599-021-00860-4

Climent, A. M., \& Vidal, P. G. (2021). Videoling: Video as an educational purpose in grammar teaching. Tejuelo, 33, 75-102. https://doi.org/10.17398/1988-8430.33.75

Collado-Valero, J., Rodríguez-Infante, G., Romero-González, M., Gamboa-Ternero, S., Navarro-Soria, I., \& Lavigne-Cerván, R. (2021). Flipped classroom: Active methodology for sustainable learning in higher education during social distancing due to COVID-19. Sustainability (Switzerland), 13(10). https://doi. org/10.339o/su13105336

Del Valle-Ramón, D., Muñoz-Repiso, A. G. V., \& Gómez-Pablos, V. B. (2020). Aprendizaje basado en proyectos por medio de la plataforma YouTube para la enseñanza de matemáticas en Educación Primaria. Education in the Knowledge Society, 21, 1-9. https://doi.org/10.14201/eks.23523

Goian, V., Goian, O., \& Biletska, T. (2021). Management Priorities of Audiovisual Student Projects During the COVID-Crisis. International Journal of Emerging Technologies in Learning, 16(10), 35-53. https://doi. org/10.3991/ijet.v16i10.19679

Hernández, J. P., \& Martinez, F. (2021). El empleo de videotutoriales en la era post COVID19: valoración e influencia en la identidad docente del futuro profesional. Revista de Educación a Distancia, 21(65), 1-18. https://doi.org/10.6018/red.449321 
Janssen, C. H. C. (2020). The flipped classroom during COVID-19. Educacion Quimica, 31(5), 173-178. https:// doi.org/10.22201/fq.18708404e.2020.5.77288

Lozano-Blasco, R., Quilez-Robres, A., Delgado-Bujedo, D., \& Latorre-Martínez, M. P. (2021). YouTube's growth in use among children 0-5 during COVID19: The Occidental European case. Technology in Society, 66, 101648. https://doi.org/10.1016/j.techsoc.2021.101648

Maraza-Quispe, B., Alejandro-Oviedo, Fernández-Gambarini, W., Cisneros-Chavez, B., \& ChoquehuancaQuispe,W. (2020). Análisis de YouTube como herramienta de investigación documental en estudiantes de educación superior. Publicaciones de La Facultad de Educacion y Humanidades Del Campus de Melilla, 50(2), 133-147. https://doi.org/10.30827/publicaciones.v50i2.13949

Marcos Ramos, M., \& Moreno Méndez, M. (2020). La influencia de los recursos audiovisuales para el aprendizaje autónomo en el aula. Disertaciones: Anuario Electrónico de Estudios En Comunicación Social, 13(1), 97-117. https://doi.org/10.12804/revistas.urosario.edu.co/disertaciones/a.7310

Neumann, M. M., \& Herodotou, C. (2020). Evaluating YouTube videos for young children. Education and Information Technologies, 25, 4459-4475. https://doi.org/10.1007/s10639-020-10183-7

Pattier, M. D. (2020). Mirando Al Futuro: Cómo Influir En Educación a Través De Un Canal De Youtube. Revista Tecnología Educativa, 5(1), 85-94. https://tecedu.uho.edu.cu/index.php/tecedu/article/view/209

Ramírez, M. G., Rojas, R. G. de T., \& Martínez, A. A. (2020). YouTube y coronavirus: análisis del consumo de vídeos sobre la pandemia COVID-19. Revista Latina de Comunicacion Social, (78), 121-153. https://doi. org/10.4185/RLCS-2020-1471

Salas-Rueda, R. A. (2021). Impacto del aula invertida en el proceso de enseñanza-aprendizaje sobre los mapas de Karnaugh. Revista Electrónica Educare, 25(2), 1-22. https://doi.org/10.15359/ree.25-2.14

Sobeida, L., \& Navarro, S. (2021). La estrategia "Aprendo en casa” y los retos en la educación virtual peruana. EduSol, 21(76), 202-214. https://edusol.cug.co.cu/index.php/EduSol/article/view/1370

Sun, T., \& Zhong, B. (2020). Multitasking as multisensory behavior: Revisiting media multitasking in the perspective of media ecology theory. Computers in Human Behavior, 104(May 2019), 106151. https://doi. org/10.1016/j.chb.2019.09.027

Temban, M. M., Hua, T. K., \& Said, N. E. M. (2021). Exploring informal learning opportunities via youtube kids among children during COVID-19. Academic Journal of Interdisciplinary Studies, 10(3), 272-287. https:// doi.org/10.36941/AJIS-2021-0083

Triviño-Cabrera, L., Chaves-Guerrero, E. I., \& Alejo-Lozano, L. (2021). The figure of the teacher-prosumer for the development of an innovative, sustainable, and committed education in times of COVID-19. Sustainability (Switzerland), 13(3), 1-18. https://doi.org/10.3390/su13031128

Vasilchenko, A., Cajander, A., \& Daniels, M. (2020, june 14-18). Students as Prosumers: Learning from PeerProduced Materials in a Computing Science Course. [Proceedings] Frontiers in Education Conference, Uppsala, Sweden https://doi.org/10.1109/FIE44824.2020.9274042

\section{AUTORES}

Luis Alberto Chávez Ramos. Docente universitario en la Universidad particular César Vallejo y en la Universidad Nacional San Cristóbal de Huamanga. Maestro en Ciencias de la Comunicación.

Adelaida Maria del Carmen Hualpa Flores. Docente de la Universidad particular Autónoma del Perú. Maestra en Gestión de los Servicios de la Salud y Licenciada en Psicología.

Eva Luis Paredes. Magister en Educación con mención en Docencia y Gestión Educativa por la Universidad Cesar Vallejo. Licenciada en Educación Especialidad Artes - Literatura.

Edgar Hugo Vásquez Condezo. Maestro en Administración Educativa. Profesor de Educación Primaria. Especialista de Educación Básica Regular del nivel primaria en la UGEL Pasco. 